\title{
Working with ELSA - How an Emotional Support Agent Builds Trust in Virtual Teams
}

\author{
Lennart Hofeditz \\ University of Duisburg- \\ Essen, Germany \\ lennart.hofeditz@uni- \\ due.de
}

\author{
Mareen Harbring \\ University of Duisburg- \\ Essen, Germany \\ mareen.harbring@stud.uni- \\ due.de
}

\author{
Milad Mirbabaie \\ Paderborn University, \\ Germany \\ milad.mirbabaie@uni- \\ paderborn.de
}

\author{
Stefan Stieglitz \\ University of Duisburg- \\ Essen, Germany \\ stefan.stieglitz@uni- \\ due.de
}

\begin{abstract}
Virtual collaboration is an increasing part of daily life for many employees. Despite many advantages, however, virtual collaborative work can lead to a lack of trust among virtual team members, e.g., due to spatial separation and little social interaction. Previous findings indicated that emotional support provided by a conversational agent (CA) can impact human-agent trust and the perceived social presence. We developed an emotional support agent called ELSA and conducted a between-subject online experiment to examine how CAs can provide emotional support in order to increase the level of trust among colleagues in virtual teams. We found that human-agent trust positively influences the level of calculus-based trust among team members and increases team cohesion, whereas perceived anthropomorphism and social presence towards a CA seems to be less important for trust among team members.
\end{abstract}

\section{Introduction}

Even before the challenges faced by enterprises through Covid-19, widely distributed teams and projects carried out in virtual collaboration were increasingly part of the everyday life of employees [1]. In order to secure competitive advantages, it is of great importance for organizations to find ways to improve teamwork, especially within the growing field of virtual work. Despite the various advantages such as being independent of the employee's location, virtual collaboration can lead to feelings of isolation by lacking consistent physical encounters with other team members and a lack of trust among team members [2]. One reason can be the spatial separation of colleagues, decreasing the transparency of how much effort each team member puts into a project [3]. In case of new composed work teams, building trust in a virtual team is even more difficult. To increase the level of trust in virtual teams, information and communication technology (ICT) focusing on the graphical visualization of activities and work contributions of various team members can be applied [4]. Real trust in terms of equal trust that is established in physical faceto-face teamwork, however, is best built through interaction with a real person [5].

There is one specific type of ICT that represents a natural connection of both an almost real-word type of human-like trust and an appropriate virtual solution that is expected to enable a support of virtually builtup trust: a Conversational Agent (CA). CAs are automated tools that can contain human characteristics and behavioral aspects, reaching from superficial appearances to certain language features [6]. They represent a range of technologies and are designed for processing natural language that enables them to serve as a non-human interaction partner for a human user. CAs can be considered both as a teammate in virtual collaboration and as a tool for information and communication [7]. CAs (such as chatbots for strengthening mental health) are able to provide emotional support to humans by analyzing people's emotions or suggesting exercises and tasks [2]. They are also found to enrich efficient team interactions for collaboration purposes [8]. Thus, they represent a promising approach to address the problem of isolation and lack of trust within virtual teams. While there has been research on how to achieve humanagent trust, there is limited research that contributes to building trust among human virtual team members through CAs providing emotional support. In order to provide more insight into this research field, we aimed to address the issue of isolation and a lack of trust in virtual teams by raising the following research question (RQ):

How can CAs provide individual emotional support in order to increase trust among human team members within virtual collaboration? 
Based on a throughout literature analysis, we proposed a new research model for emotional support through a $\mathrm{CA}$ in a virtual work context and developed an emotionally supportive CA, called ELSA (EmotionaL Support Agent). ELSA can provide emotional support to individual team members during work related tasks. We evaluated ELSA in a betweensubject online experiment. One group received emotional support during a work-related task through ELSA and the control group only received task related support by a CA.

With this work we provide knowledge on how CAs can be applied not only as a useful tool that provides work related information, but also as virtual teammate that spends emotional support in order to increase trust and team cohesion within virtual teams.

\section{Related Work}

There is a wide range of collaboration technologies which can be applied to support virtual collaborative work such as virtual assistants, CAs or other AI-based collaboration technology [9]. The progress of these collaborative technologies suggests that in the future, these machines will be more than tools that support team performance. Mirbabaie et al. [7] were already able to show that these technologies can be perceived as both supportive collaboration tools and virtual teammates. This offers a variety of new opportunities to mitigate shortcomings of virtual collaboration.

One of the most widely researched and deployed collaboration technologies in virtual collaboration are CAs [9, 10, 11, 12, 13]. A CA can be defined as "software that interacts and exchanges information with its users through natural language" [8:1] by using natural language processing. Despite application fields like customer service and education, they can also be used for organizational purposes such as task management in in virtual collaborative work [14]. For CAs to be adopted and used by employees, it is necessary to ensure that CAs are perceived trustworthy [15]. Trust in a CA is found to have a positive effect on its acceptance of a human user [15], creating positive experiences and establishing a stable relationship with it [16]. Furthermore, trust in a CA has a positive effect on the intention to use it again in the future [17]. For the present context, we consider trust in a CA as "an individual's belief in the competence, dependability and security" of a CA [18:482].

Team members of conventional face-to-face teams also establish trustworthiness through personal and social cues [3]. Resulting from a systematic literature review the taxonomy of Feine et al. [19] provides a comprehensive and up-to-date overview of social cues for CAs, summarized from different contexts and terminologies of empirical literature. They define the term social cue as "a cue that triggers a social reaction towards the emitter of the cue" [19:11]. According to this definition, a social cue develops into a social signal which is perceived by a human user. This in turn triggers a social reaction towards a CA. The taxonomy comprises a total of 48 social cues which can be divided into four main categories with several corresponding sub-categories. The main categories are verbal, visual, auditory and invisible. Social cues provided by people in a physical face-to-face encounter are barely given within interaction of virtual teams. They are easily lost or misinterpreted via digital communication. Therefore, we implemented social cues in ELSA's vocabulary and appearance to enrich the virtual collaboration by transmitting social cues during each team member's individual conversation. Therefore, we support the building of trust among team members as well as trust towards the CA.

As we found that, in addition to social cues, emotional support though a CA can also result in team cohesion and trust among virtual team member, we focused on socio-emotional CA behavior. Janssen et al. [20] define the term of socio-emotional behavior as an ability of a CA to have a conversation with the awareness of what topic is being discussed. This includes showing empathy for the user's individual needs as well as responding to expressed emotions of the human user. Emotions are human characteristics that a technology like a CA does not naturally possess. In order to provide emotional support, a CA needs to contain human-like emotional characteristics such as anthropomorphic features which can be summarized as "the attribution of human-like physical or nonphysical features, behavior, emotions, characteristics and attributes to a non-human agent" [10:523]. Anthropomorphic features are found to have a significant impact on the way users perceive the interaction with a CA and are closely connected to social cues [19]. In addition, they can have a positive impact on the believability of a CA [6], on the desire to interact with it [21] and on a CA's social presence [22]. Social presence represents the emotional component of anthropomorphism. It relates to the aspects of empathy, sociability and warmth of human behavior [23]. Social presence of a CA is found to predict the acceptance of it [24]. The implementation of social and anthropomorphic cues also leads to the well-known Computers are Social Actors (CASA) paradigm $[25,26]$ which states that humans behave towards computers in the same way as towards other humans, although the human user is aware of the fact 
that a computer is no human being. Although the relationship of social presence, social cues, and anthropomorphism with trust is already suggested, it is largely unclear how CAs can use targeted emotional support to improve trust within virtual teams. What is clear, however, is that there is often a lack of resources for providing emotional support that can be addressed by applying CAs [2].

\section{Theoretical Background}

An overview of the empirical literature reveals that there is no common agreement on a uniform definition for the construct of trust due to its interdisciplinarity nature. According to Lewicki and Bunker [5], we adopt the definition of Boon and Holmes [27], as it covers the crucial elements of a multitude of definitions. Accordingly, trust is defined as " a state involving confident positive expectations about another's motives with respect to oneself in situations entailing risk" [27:194].

Although some empirical trust models assume that trust can only develop over time, there also exists the paradox of high initial trust levels which demonstrates a high level of trust after a short time [28]. We decided to focus on this high initial trust levels, as we assumed that emotional support is especially necessary for employees in their initial stage of virtual collaborative work in order to achieve a high level of trust within a team. The High-Level Model of Initial Formation of Trust aims to explain the process of building initial trust in an organizational context [28]. Overall, there is a consensus in research that the level of trust increases in the form of successive stages. A three-part division into the successive levels of a building stage, followed by a stabilization stage and ending in a mature state of trust, often appears in the literature [29].

Against the background of the High-Level Model of Initial Formation of Trust, Lewicki and Bunker's model of organizational trust building [5] serves as the underlying theoretical basis to define the understanding of trust in this work. Lewicki and Bunker [5] propose three types of trust: calculus-based trust, knowledge-based trust and identification-based trust. Calculus-based trust refers to "consistency of behavior" [5:118], whether a trustee really does what he or she says. This stage represents the early, initial formation of trust and therefore serves as the focus of this research. People in this phase have not yet had any background information or past experience that they can use to form an opinion about a trustee's trustworthiness. This stage is therefore mainly about weighing up costs and benefits by entering into a trusting relationship. A risk factor of whether a trustee fulfills the expectations placed on him or her also plays a role here as to whether a trustor considers it beneficial to trust the trustee [30].

Trust building has already been widely examined in the context of virtual collaboration with employees working spatially distributed and can be considered as considered crucial for virtual collaboration. Pinsonnault and Caya [31] state that "trust is one of the most important process variables in virtual team research" [31:4]. In virtual teams, trust needs to replace the supervision and transparency of the work of other team members [3]. For virtual teams, building trust among one another is a much greater challenge than for conventional teams mainly because of the limited personal contact.

Existing trust within a team can have a positive impact on other aspects of group dynamics like team cohesion. Team cohesion was found to be the "most important small group variable" [32:259] and can be defined as the degree to which team members like each other and desire to remain in the team [33]. Trust in other team members and team cohesion show a significant relationship and have frequently been examined together. Accordingly, a high level of trust within a team could lead to a higher level of team cohesion.

Working in virtual teams can also have an impact on the well-being, "defined as the physical and mental health of employees" [34:363]. Thus, a connection between remote workers and low workplace wellbeing can be assumed in the manner of psychological, social and physical points of view. Besides social contact, trust at the workplace can be a fundamental predictor for the construct of subjective well-being (SWB). Considering these related works and background, we derived the following nine hypotheses:

H1: Receiving emotional support from a $C A$ in virtual collaboration positively affects the level of calculusbased trust in the team.

H2: Receiving emotional support from a CA in virtual collaboration positively affects the level of humanagent trust.

H3: Receiving emotional support from a $C A$ in virtual collaboration positively affects the level of perceived anthropomorphism.

H4: Receiving emotional support from a $C A$ in virtual collaboration positively affects the level of perceived social presence.

H5: Human-agent trust has a positive impact on the level of calculus-based trust in virtual collaboration.

H6: Perceived anthropomorphism has a positive impact on the level of calculus-based trust in virtual collaboration. 
H7: Perceived social presence has a positive impact on the level of calculus-based trust in virtual collaboration.

H8: Calculus-based trust among team members in virtual collaboration has a positive impact on the level of team cohesion.

H9: Calculus-based trust among team members in virtual collaboration has a positive impact on the level of $S W B$.

H10: Human-agent trust has a positive impact on the level of team cohesion.

\section{Research Design}

\subsection{CA Design and Procedure}

To examine the proposed model we designed ELSA, a CA with consideration of emotional support, containing trustworthy, social, anthropomorphic and trust supporting cues. Regarding a between-subject design and in order to examine ELSA's effectiveness, we also developed a second CA named TaskBot. Contrary to ELSA, TaskBot exclusively interacts on a task-related basis and its design of communication did not include any kind of social cues and emotionally supporting phrases. We provide an overview of ELSA's cues in Table 1.

Table 1. Social, anthropomorphic, trustworthy and trust building cues of ELSA

\begin{tabular}{|l|l|}
\hline Category & Examples \\
\hline $\begin{array}{l}\text { Social cues } \\
\text { (Feine et al. [6]) }\end{array}$ & $\begin{array}{l}\text { Greetings, thanking, small talk, } \\
\text { smileys \& informal language }\end{array}$ \\
\hline $\begin{array}{l}\text { Anthropomorph } \\
\text { ic cues (Peuffer } \\
\text { et al. [10]; [35]) }\end{array}$ & $\begin{array}{l}\text { Profile picture, delayed } \\
\text { response time, and referring to } \\
\text { herself as "I" }\end{array}$ \\
\hline $\begin{array}{l}\text { Cues for } \\
\text { trustworthiness } \\
\text { towards CA [15, } \\
17]\end{array}$ & $\begin{array}{l}\text { Giving transparency \& using } \\
\text { an anthropomorphic verbal } \\
\text { style }\end{array}$ \\
\hline $\begin{array}{l}\text { Cues for } \\
\text { providing } \\
\text { emotional } \\
\text { support }\end{array}$ & $\begin{array}{l}\text { Providing feedback about the } \\
\text { team's work progress and } \\
\text { asking about the impressions } \\
\text { about the other team members }\end{array}$ \\
\hline
\end{tabular}

The two CAs only differed in the way of how communication was controlled. ELSA was controlled according to the Wizard of $\mathrm{Oz}$ method which is a wellknown technique for research in the context of humancomputer interaction [36]. ELSA's responses were controlled by an experimenter in order to simulate the realistic functioning of ELSA as a CA, while the participants were not informed about the fact that
ELSA was controlled by a human. Nevertheless, it was clearly communicated in advance that ELSA is an automated CA. On the other side, TaskBot was implemented and realized using Dialogflow from Google. However, for the participants, the difference was not visible as both groups interacted with the CA in the same interface via Slack.

To test ELSAs abilities, we conducted an online team experiment via Zoom and Slack. The evaluation contained an interaction with ELSA or TaskBot followed by subsequent questionnaires about the interaction and perception. We chose a text-based CA as because it represents the most commonly used type of CAs in practice. To simulate a realistic organizational environment, both ELSA and TaskBot were implemented in the instant messenger software Slack which is often used for organizational team communication. Participants were recruited online via social network sites. The assignment of the participants to one of the two conditions was conducted randomly.

During the first part of the evaluation, a virtual work team consisting of three to five participants was invited to a Zoom room to get to know each other before each of the team members interacted with one of the CAs. Each participant received a link to LimeSurvey, in which demographic data and information about previous experience with CAs were inquired. Afterwards, the participants receive further instructions for the subsequent individual CA interaction and the access data for Slack. The participants were asked to solve the Desert Survival Task - which is a widely known team building task with the advice to contact their CA for help. After solving the task and interacting with the CAs in a separate meeting room, participants of both groups were asked to answer questionnaires on the interaction with the CA and their perception of the virtual team. This part is expected to show whether participants who interacted with ELSA are able to establish a higher level of calculus-based trust with one another. The data collection period is from 11th of December 2020 to 31 st of January 2021. In Figure 1, we provide a representative screenshot of how our participants interacted with ELSA.

\subsection{Questionnaires}

Our survey consisted of a total of ten different questionnaires. We used Likert scales reaching from 1 (strongly disagree) to 7 (strongly agree). At the 


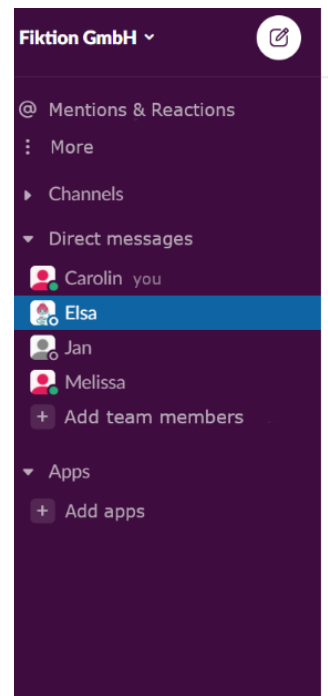

Carolin $10: 41$ AM
Hello
Elsa $10: 42$ AM
Hi, glad to have you here! I would like to briefly introduce myself: My name is Elsa. I am here to support you in your com-
mon task $\because$ Through my time here at the company, I have already been able to gain a lot of experience in teamwork.
What's your name?
Carolin 10:42 AM
Carolin
Elsa 10:42 AM
I am glad to meet you, Carolin $:$
I think it's really cold today! How is the weather today with you?
Carolin 10:42 AM
Also very cold and wet
Elsa 10:42 AM
I heard you already met your other team members Jan-Sebastian and Melissa. Did you have a good first
impression?
Carolin 10:42 AM
Yes, they both seem nice $:$

Figure 1. Representative extract from a participant's conversation with ELSA

beginning, demographic information of the participants, namely age, gender, level of education and level of occupation were requested. We measured previous experience with CAs by using the subconstruct Experience of the Use of Technology questionnaire [37]. This was followed by the questionnaire on the construct of human-agent trust, adapted from the original Human-Computer Trust questionnaire [38]. For reasons of content fitting, we only adopted the sub-constructs Benevolence and Reciprocity. The level of team cohesion was measured by Seashore's [39] Index of Group Cohesiveness. The level of calculus-based trust among each team member was measured by the Calculus-Based Trust Scale [30]. To measure how human-like the CAs were perceived we measured anthropomorphism [35]. Our questionnaire on social presence [40] consisted of five items in total. In addition, we measured SWB according to Ashleigh et al. [34] by the Subjective Happiness Scale [41] and the Satisfaction with Life Scale [42]. The penultimate block of questions comprised six items on the future use of the CA. With a self-developed item, we questioned whether the participant would like to use such an CA as ELSA or the TaskBot for his or her (future) job. Lastly, the question block included the sub-construct Task Fit from the Use of Technology questionnaire [37].

\subsection{Sample}

A total of 98 participants, of which 96 were considered as valid data sets, took part in our survey. Two participants have to be excluded, as they failed two attention check questions. Participants of the final sample were between 18 and 47 years old, with an average age of 22 years. The sample consists of 73 female (76.0\%) and 22 male (22.9\%) participants. One participant gave the answer "diverse". 22 people stated that they had minor difficulties with the CA or that the CA did not always provided correct answers. On average, the participants had little previous experience with CAs $(M=2.82)$. In this respect, the groups of ELSA and TaskBot did not differ significantly. We found a distribution across the group conditions of 47 participants in the experimental group $(n=47)$ and 49 participants in the control group $(n=49)$.

\section{Results}

\subsection{Statistical Results}

For examining the stated hypotheses of the proposed research model, we calculated one-way analyzes of variance (H1-4) and linear regressions (H5-9) using IBM SPSS version 27. In addition to validity, the reliability of all constructs is also examined in advance. The reliability shows an acceptable value for each construct since the limit of .7 is exceeded for all of them.

We first checked the prerequisites for conducting all following one-way analyzes of variance. The requirement of normal distribution was not met for $\mathrm{H} 1$ and $\mathrm{H} 3$. We summarized the results of our calculations for H1-4 in Table 2.

\section{Table 2. One-way analyses of variance}

\begin{tabular}{|c|c|c|c|c|c|}
\hline & $\boldsymbol{F ~ ( 1 , 9 4 ) ~}$ & $\boldsymbol{p}$ & $\boldsymbol{\eta}^{\mathbf{2}}$ & $\boldsymbol{M}_{\text {ELSA }}$ & $\boldsymbol{M}_{\text {TaskBot }}$ \\
\hline H1 & 3.92 & .051 & .04 & 4.85 & 4.35 \\
\hline H2 & 12.61 & .001 & .12 & 5.12 & 4.36 \\
\hline H3 & 7.90 & .006 & .08 & 3.10 & 2.26 \\
\hline H4 & 67.80 & $<.001$ & .42 & 4.62 & 2.38 \\
\hline
\end{tabular}


To examine the influences of human-agent trust (H5), perceived anthropomorphism (H6) and perceived social presence $(\mathrm{H} 7)$ on the level of calculation-based trust, we calculated linear regressions.

The fulfillment of prerequisites for calculating a linear regression analysis were checked in advance. The results of the regression for H5 showed that the level of human-agent trust could significantly predict the level of calculus-based trust with a positive, moderate effect size (see Table 3). According to Cohen [43], this represents a moderate effect size with $f^{2}=.20$. With regard to the assumed causal relationship of H6, we found that the requirement for a linear relationship of perceived anthropomorphism and calculus-based trust was not met. For perceived social presence and calculus-based trust $(\mathrm{H} 7)$ the requirement of a linear relationship was also violated. We therefore did not further carry out the other linear regressions.

Table 3. Overview of linear regressions

\begin{tabular}{|c|c|c|c|c|c|}
\hline & $\boldsymbol{\beta}$ & $\boldsymbol{t}(\mathbf{4 5})$ & $\boldsymbol{p}$ & $\boldsymbol{R}^{\mathbf{2}}$ & $\boldsymbol{F}(\mathbf{1}, \mathbf{4 5})$ \\
\hline H5 & .41 & 3.03 & .004 & .17 & 9.15 \\
\hline H8 & .49 & 3.81 & .001 & .24 & 14.54 \\
\hline
\end{tabular}

Furthermore, we calculated a linear regression to test H8. The results showed that the level of calculusbased trust could significantly predict the level of team cohesion with positive, moderate influence (see Table 3). The effect size was medium to rather high. Accordingly, the size of $f^{2}$ amounts .32 which, according to Cohen [43], could be classified as a medium to large effect size. The requirement of a linear relationship was violated for calculus-based trust and SWB (H9), resulting in that we did not carried out a further linear regression.

As the results reveal a significant positive causal relation between human-agent trust and the level of calculus-based trust, we also assumed an influence of human-agent trust on team cohesion (H10). The results of the respective linear regressions showed that the level of human-agent trust could significantly predict the level of team cohesion with a high positive influence $(\beta=.57, t(45)=4.62, p<.001)$. The level of human-agent trust could also explain $32.2 \%$ significant proportion of the total variance in the team cohesion level $\left(R^{2}=.32, F(1,45)=21.35, p<.001\right)$. With $R^{2}=.32$, the linear model fitted the data well and, according to Cohen [43], represented a very large effect size $\left(f^{2}=.47\right)$. We summarized our results in Figure 2.

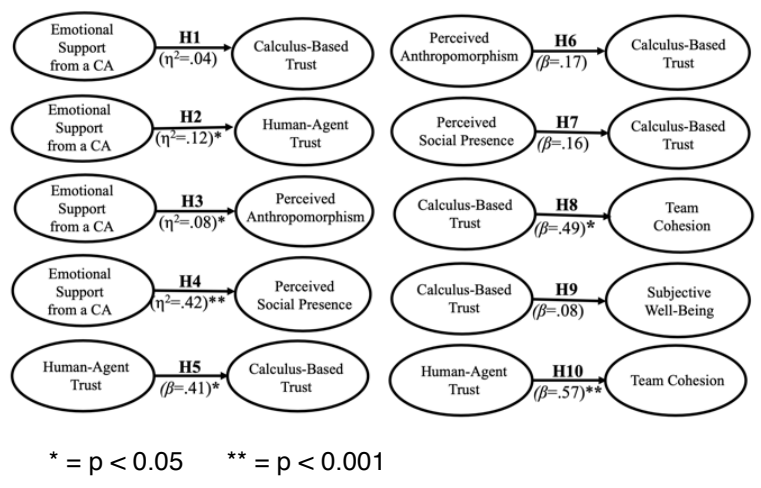

Figure 2. Visualization of the main findings

\subsection{Further Results}

In addition to these results, we found further relevant aspects that we recognized through the evaluation of ELSA and TaskBot. Participants who interacted with ELSA used more emoticons overall, chose a nicer tone of texting and showed interest in ELSA counter-questions from the participants. In addition, the task fit was also requested in the survey, that is, the extent to which the participants believed that the future use of the respective CA would also be well suited for future tasks. The experimental group that interacted with ELSA showed a higher mean value $\left(M_{E L S A}=4.29\right)$ than the control group $\left(M_{\text {TaskBot }}=\right.$ 3.78). When they were asked whether they would like such a CA as they got to know in the experiment for their job, the majority of the experimental condition answered with "yes" $(n=31)$, while the majority of the participants from the control answered with "no" $(n=31)$.

\section{Discussion}

We expected to find a difference in the calculusbased trust level of participants between interacting with ELSA and TaskBot. However, our findings did not support this hypothesis. Thus, we cannot conclude that emotional support of a CA can directly contribute to a higher level of calculus-based trust among team members (H1 not supported). According to the mental model approach [44], one possible explanation is that ELSA possibly was still perceived as too robotic which resulted in an aversion for interacting with her. In connection with human/CA perception, the phenomenon of Uncanny Valley [45] could be another explanation. The low average value of previous experience with CAs of the sample suggests that it takes more time for unexperienced users to get used to a CA interaction. However, arguments stating that it would be easy for teams to build trust in the initial 
phase of teamwork [28] and that teams are also able to build this up quickly, are not to be completely invalidated by the result of H1. Since the initial phase of teamwork, to which the measurement of calculusbased trust relates, is not limited to one initial task, it is entirely possible that a longer lasting experiment could bear obverse findings.

The statistical result of $\mathrm{H} 2$ supports that the groups of ELSA and TaskBot differ significantly in their level of trust towards the respective CA. This finding confirmed previous literature on anthropomorphic and social cues, as these cues are considered to have a positive influence on believability and trust in a CA [23]. Although trust is a phenomenon that originally refers to be developed between humans, trustworthy cues, in connection with social and anthropomorphic cues, can certainly contribute to the fact that non-human technologies like CAs can be classified as trustworthy.

H5 was supported by the result of the linear regression. It can be deviated that the design of a trustsupporting CA should not be limited to anthropomorphic and social cues, but rather need to include trustworthy cues (e.g., transparency about what other team members do or trust towards the CA). This finding can be explained by the fact that people are more likely to engage trust-supporting communication measures if they consider a CA trustworthy. Consistent with the work of [46], this was reached by reducing uncertainty towards ELSA.

For H3, the conducted calculation showed the expected significant difference between the conditions with regard to perceived anthropomorphism (H3 supported). The finding also confirmed previous research on anthropomorphic cues, especially a correspondence with the CASA paradigm [26] can be recognized here. The aspect of social presence may also have contributed to the result of the significantly higher mean value for the experimental group, since aspects such as human warmth are considered to be the social component of anthropomorphic cues [23].

Contrary to our expectations, H6 could not be supported by or results. An insufficient level of perceived anthropomorphism could be responsible for the fact that the corresponding technology is not accepted by a human, or in the present case not sufficiently accepted for effective emotional support. At this point, the Uncanny Valley approach [45] can be considered again for explanation, since the mean value for the experimental group shows a rather medium value of perceived anthropomorphism. As another explanation, it may not be necessary to design a CA as human as possible for the purpose of emotional support. According to [21], "learning purely from human-human behavior may not always be the most effective approach" (p. 74). This can also be supported by the fact that most of our participants stated that they would like to use ELSA for their work.

For H4, we found a significant difference between the group conditions with regard to the perceived social presence of the respective CA. Accordingly, the group's mean values for the construct of social presence showed that the social presence of ELSA was perceived as much higher than for TaskBot. The result fit with previous research that concluded that anthropomorphic cues have a positive effect on the perceived social presence $[23,47]$. According to the CASA paradigm [26], the finding suggests that it is entirely possible to attribute socio-emotional characteristics such as human warmth, empathy and sociability to a CA.

With regard to social presence, we assumed that perceived social presence is suitable for predicting the calculus-based trust level among team members who receive emotional support. However, our results showed that $\mathrm{H} 7$ could not be supported. This result differs from previous studies which found social presence as a suitable predictor for trust especially in the context with CAs [48]. Despite the short interaction time, it has been shown that calculus-based trust has a significantly positive effect on team cohesion in the present work. Thus, the assumption of H8 was supported. Although the groups of experimental and control condition do not significantly differ in terms of their level of calculusbased trust, it is still evident, that trust and team cohesion are positively related and that trust can serve as suitable predictor for predicting team cohesion [32].

As H9 was not supported, we assume no effect for the causal relationship of calculus-based trust as a predictor and the criterion SWB. Against previous research suggesting a relationship between existing trust in work colleagues and SWB [34], SWB can neither be supported nor transferred to the construct of calculus-based trust.

As a further result, the response behavior of participants towards both CAs was conspicuous. The majority of participants interacting with ELSA behaved very nicely, sensitively and with interest towards the CA while this was not the case towards TaskBot. This phenomenon supports the CASA paradigm [26]. Although ELSA's focus lies on socioemotional behavior rather than efficiency, the result suggest that the two aspects of socio-emotional interaction and efficiency do not seem to contradict each other. Despite small talk and other non-task related questions, the participants interacting with ELSA indicated that they would like to use the system for future tasks in their everyday work. The finding fits with empirical literature that states "trust in [virtual] 
advisors positively affect their reuse intentions" [17:3]. It can be derived from this finding that social talk is at least as important to users as efficiency.

The investigated influence of human-agent trust on team cohesion showed a significant effect (H10 supported). We conclude that trust is a suitable predictor for team cohesion. Against this background, it can be deduced as an important finding of this work that both kinds of trust (calculus-based trust towards other human team members and trust in a CA) can predict the level of team cohesion supported by emotional support. In contrast, the exploratory investigated influence of human-agent trust on SWB shows no significant causal relationship. It can be deduced that both types of trust - calculus-based trust and trust in a CA - do not influence the level of a user's SWB in the initial phase of virtual teamwork. Although the mean value of human-agent trust towards ELSA was significantly higher than the mean value of team trust, in general, a high level of trust does not have any significant influence on SWB in the present context.

\subsection{Implications}

With this work, we contribute to research and practice. The paradox of high initial levels of trust in new formed teams stated by [38] can be claimed as transferable to a certain extent to the construct of calculus-based trust in emotional supported virtual collaboration because there is a medium-high entry level of calculus-based trust already with the first interaction to be observed. It can be derived that a CA's emotional support is more effective in teams with a high tendency to trust technologies such as CAs. In order to be able to transfer the initial phase of Lewicki and Bunker's [5] trust model to virtual collaboration supported by a CA, it needs to be taken into account that the design of an emotional support agent should contain trustworthy cues. The high-level model of initial formation of trust [28] can be used as a starting point to understand emotional support by a CA to increase the level of trust in virtual teams. In addition, the result of the statistical examination of $\mathrm{H} 9$ concerning the causal relationship of calculus-based trust on SWB contributes additional knowledge to the question of how mental health of employees who work in virtual collaborative teams can be improved.

If organizations decide to integrate an emotional support agent into their work routine, they can benefit from the present results by adopting ELSA's cues for being considered as a trustworthy CA. From an employee's point of view, we provide evidence that socio-emotional communication is at least as important to users as efficiency which should be taken into account by organizations. We also could show that employees stated that they would like to further use ELSA for their own work.

\subsection{Limitations and Further Research}

This article comes with some limitations and suggestions for future studies. For the present research, we focused on emotional support for newly assembled work teams without a common background and thus, exclusively on the first stage of building trust. Therefore, researchers could extend the present findings by developing an emotional support agent for the two subsequent stages of the model of organization trust building [28]. As the trend towards virtual work increases, also through artificial intelligence, the creation of a multimodal CA and an animated version in virtual reality would also be interesting.

Due to the fact that we conducted an elaborate experiment, our sample was not as large as for an online survey and our participants were relatively young. Therefore, future research could examine larger teams in a real virtual organizational setting. In addition, the length of task could be varied. Due to the circumstances of an online experiment, internet crashes appeared in a few cases resulting in some teams consisting of only two participants.

For technical reasons, ELSA was also not able to recognize the current user's mood in real time and to adapt her response to it. It would therefore be interesting to create a CA which is able to carry out a sentiment analysis of the user's response behavior. An investigation over time with repeated measurements would also be interesting to determine a development of a participant's initial level of SWB. As globally distributed teams are not the focus of this work, the influence of cultural diversity could be added to the proposed research model. In this regard, additional attention needs to be paid to possible language barriers and cultural differences. In addition to cultural differences, the inclusion of communication theories, such as the investigation of virtual and gender communication, would also be interesting.

Finally, the aspect of gender stereotypes gets reinforced, because emotional support is more likely to be attributed to women. To counteract this, an investigation with a genderless and a masculineappearing CA would be interesting. Participants also had predominantly a female gender, so that this could have had an impact on the perception of ELSA's sympathy. 


\section{Conclusion}

With this work, we were able to show that calculus-based trust among virtual collaborative team members can be strengthen by applying a CA with trustworthy cues. Emotional support is more effective if a human classifies a CA as trustworthy. When designing an emotional support agent, researchers should consider equipping a $\mathrm{CA}$ with cues that make a CA appear trustworthy to a human user. In addition, it can be derived that emotional support of a $\mathrm{CA}$ in virtual collaboration is more effective in teams with a high tendency to trust a CA.

Our findings suggest that anthropomorphism is not the most important design feature of a CA that is aimed to possess socio-emotional behavior. We showed that it is not the purely human characteristics, but the trust-indicating cues that influence calculusbased trust. This does not indicate that anthropomorphic cues are irrelevant for supporting humans emotionally, but suggests that the main focus for future research should focus less on humanization and more on the reliable functioning of a $\mathrm{CA}$. We observed that calculus-based trust in virtual collaboration starts to build already with the first interaction, even with an average medium-high level supporting the existence for the paradox of high initial trust levels [28].

Based on the highlighted key findings, the RQ of the present work could be answered by showing how an emotional support agent can be designed to provide emotional support for individual human team members in order to increase trust within virtual collaborative teams. Because anthropomorphic and social cues presumably also contribute to a $\mathrm{CA}$ being perceived as trustworthy, this aspect should not be neglected. However, the key for providing emotional support through a CA lies in its trustworthiness.

\section{References}

[1] Hassell, M., and J. Cotton, "Some things are better left unseen: Toward more effective communication and team performance in video-mediated interactions", Computers in Human Behavior 73, 2017, pp. 200-208.

[2] Denecke, K., S. Vaaheesan, and A. Arulnathan, "A Mental Health Chatbot for Regulating Emotions (SERMO) - Concept and Usability Test", IEEE Transactions on Emerging Topics in Computing 99(1), 2020.

[3] Plotnick, L., S.R. Hiltz, and R.J. Ocker, "Trust in partially distributed teams", ICIS 2009 Proceedings Thirtieth International Conference on Information Systems, (2009), 1-17.

[4] Al-Ani, B., and D. Redmiles, "Trust in distributed teams: Support through continuous coordination", IEEE Software 26(6), 2009, pp. 35-40.
[5] Lewicki, R.J., and B.B. Bunker, "Developing and maintaining trust in work relationships", In Trust in Organizations: Frontiers of Theory and Research. SAGE Publications, Inc., 1996, 114-139.

[6] Feine, J., U. Gnewuch, S. Morana, and A. Maedche, "A Taxonomy of Social Cues for Conversational Agents", International Journal of Human Computer Studies 132(September 2018), 2019, pp. 138-161.

[7] Mirbabaie, M., S. Stieglitz, F. Brünker, L. Hofeditz, B. Ross, and N.R.J. Frick, "Understanding Collaboration with Virtual Assistants - The Role of Social Identity and the Extended Self", Business \& Information Systems Engineering Published, 2020.

[8] Diederich, S., A.B. Brendel, S. Lichtenberg, and L. Kolbe, "Design for Fast Request Fulfillment or Natural Interaction? Insights from an Experiment with a Conversational Agent", 27th European Conference on Information Systems (ECIS), (2019), 1-17.

[9] Seeber, I., E. Bittner, R.O. Briggs, et al., "Machines as teammates: A research agenda on $\mathrm{AI}$ in team

collaboration", Information and Management 57(2), 2020, pp. 103174.

[10] Pfeuffer, N., A. Benlian, H. Gimpel, and O. Hinz, "Anthropomorphic Information Systems", Business \& Information Systems Engineering 61(4), 2019, pp. 523533.

[11] Tavanapour, N., and E.A.C. Bittner, "Automated Facilitation for Idea Platforms: Design and Evaluation of a Chatbot Prototype", International Conference on Information Systems (ICIS) Proceedings, 2018, pp. 1-9. [12] Seeber, I., L. Waizenegger, S. Seidel, and S. Morana, "Reinventing Collaboration With Autonomous Technology-Based Agents", 2019.

[13] Elson, J.S., D. Derrick, and G. Ligon, "Examining Trust and Reliance in Collaborations between Humans and Automated Agents", Proceedings of the 51st Hawaii International Conference on System Sciences, 2018, pp. 430-439.

[14] Toxtli, C., A. Monroy-Hernández, and J. Cranshaw, "Understanding chatbot-mediated task management", Proceedings of the 2018 CHI Conference on Human Factors in Computing Systems, 2018, pp. 1-6.

[15] Benke, I., "Towards Design Principles for Trustworthy Affective Chatbots in Virtual Teams", ECIS 2020

Proceedings, ECIS 2020 (2020), 1-12.

[16] Benbasat, I., and W. Wang, "Trust in and adoption of online recommendation agents", ournal of the Association for Information Systems 6(3), 2005, pp. 72-101.

[17] Al-Natour, S., I. Benbasat, and R. Cenfetelli, "Trustworthy virtual advisors and enjoyable interactions: Designing for expressiveness and transparency", 18th European conference on information systems, ECIS 2010, ECIS 2010 Proceedings (2010), 1-12.

[18] Ogonowski, A., A. Montandon, E. Botha, and M. Reyneke, "Should new online stores invest in social presence elements? The effect of social presence on initial trust formation", Journal of Retailing and Consumer Services 21(4), 2014, pp. 482-491.

[19] Feine, J., U. Gnewuch, S. Morana, and A. Maedche, "A taxonomy of social cues for conversational agents", International Journal of Human-Computer Studies 132, 
2019, pp. 138-161.

[20] Janssen, A., J. Passlick, D. Rodríguez Cardona, and M.H. Breitner, "Virtual assistance in any context: A taxonomy of design elements for domain-specific chatbots", Business and Information Systems Engineering 62(3), 2020, pp. 211-225.

[21] McDuff, D., and M. Czerwinski, "Designing emotionally sentient agents", Communications of the ACM 61(12), 2018, pp. 74-83.

[22] Rietz, T., I. Benke, and A. Maedche, "The Impact of Anthropomorphic and Functional Chatbot Design Features in Enterprise Collaboration Systems on User Acceptance", 2019 14th International Conference on

Wirtschaftsinformatik (WI'19), 2019, pp. 1656-1670.

[23] Qiu, L., and I. Benbasat, "Evaluating anthropomorphic product recommendation agents: A social relationship perspective to designing information systems", Journal of Management Information Systems 25(4), 2008, pp. 145182.

[24] Benbasat, I., A. Dimoka, P.A. Pavlou, and L. Qiu, "Incorporating social presence in the design of the anthropomorphic interface of recommendation agents: Insights from an fmri study", 31st International Conference on Information Systems, ICIS 2010 Proceedings (2010), 122.

[25] Lee, J.-E.R., and C.I. Nass, "Trust in computers: The computers-are-social-actors (CASA) paradigm and trustworthiness perception in human-computer communication", In Trust and technology in a ubiquitous modern environment: Theoretical and methodological perspectives. IGI Global, 2010, 1-15.

[26] Reeves, B., and C. Nass, The media equation: How people treat computers, television, and new media like real people, Cambridge University Press, Cambridge, UK, 1996.

[27] Boon, S.D., and J.G. Holmes, "The dynamics of interpersonal trust: Resolving uncertainty in the face of risk", In Cooperation and prosocial behavior. Cambridge University Press, 1991, 190-211.

[28] McKnight, D.H., L.L. Cummings, and N.L. Chervany, "Initial trust formation in new organizational relationships", The Academy of Management Review 23(3), 1998, pp. 473-490.

[29] Siakas, K. V, D. Maoutsidis, and E. Siakas, "Trust facilitating good software outsourcing relationships", In European Conference on Software Process Improvement. Springer, Berlin, Heidelberg, 2006, 171-182.

[30] Zhao, N., Y. Shi, Z. Xin, and J. Zhang, "The impact of traditionality/modernity on identification- and calculusbased trust", International Journal of Psychology 54(2), 2019, pp. 237-246.

[31] Pinsonneault, A., and O. Caya, "Virtual teams: What we know, what we don't know", International Journal of eCollaboration 1(3), 2005, pp. 1-16.

[32] Liu, Y.C., C. Lin, and Y.A. Huang, "How do virtual teams work - A social relationship model by SEM", Proceedings of the International Conference on Electronic Business, (2007), 258-260.

[33] Huang, R., T. Carte, and L. Chidambaram, "Cohesion and performance in virtual teams: An empirical investigation", Proceedings of the Tenth Americas
Conference on Information Systems, (2004), 1283-1290. [34] Ashleigh, M.J., M. Higgs, and V. Dulewicz, "A new propensity to trust scale and its relationship with individual well-being: Implications for HRM policies and practices", Human resource management journal 22(4), 2012, pp. 360-376.

[35] Seeger, A., J. Pfeiffer, and A. Heinzl, "Designing Anthropomorphic Conversational Agents : Development and Empirical Evalua- tion of a Design Framework", Thirty Ninth International Conference on Information Systems, AIS (2018), 1-17.

[36] Riek, L.D., "Wizard of Oz studies in HRI: A systematic review and new reporting guidelines", Journal of Human-Robot Interaction 1(1), 2012, pp. 119-136. [37] Thompson, R.L., C.A. Higgins, and J.M. Howell, "Influence of experience on personal computer utilization: Testing a conceptual model", Journal of Management Information Systems 11(1), 1994, pp. 167-187.

[38] Gulati, S.N., S.C. Sousa, and D. Lamas, "Design, development and evaluation of a human-computer trust scale", Behaviour and Information Technology 38(10), 2019, pp. 1004-1015.

[39] Seashore, S.E. 1979., Group cohesiveness in industrial work groups, University of Michigan, Ann Arbor, Michigan, USA, 1954.

[40] Gefen, D., and D. Straub, "Managing user trust in B2C e-services", e-Service Journal 2(2), 2003, pp. 7-24. [41] Lyubomirsky, S., and L. Ross, "Changes in attractiveness of elected, rejected, and precluded alternatives: A comparison of happy and unhappy individuals", Journal of Personality and Social Psychology 76(6), 1999, pp. 988-1007.

[42] Diener, E., R.A. Emmons, R.J. Larsen, and S. Griffin, "The satisfaction with life scale", Journal of Personality Assessment 49(1), 1985, pp. 71-75.

[43] Cohen, J., Statistical Power Analysis for the Behavioral Sciences, Taylor and Francis, Hoboken, 1988. [44] Feng, S., and P. Buxmann, "My virtual colleague: A state-of-the-Art analysis of conversational agents for the workplace", Proceedings of the 53rd Hawaii International Conference on System Sciences, (2020), 156-165.

[45] Mori, M., “The Uncanny Valley”, Energy 7(4), 1970, pp. 33-35.

[46] Söllner, M., A. Hoffmann, H. Hoffmann, A. Wacker, and J.M. Leimeister, "Understanding the formation of trust in IT artifacts", 33rd International Conference on Information Systems, Association for Information Systems (2012).

[47] Rietz, T., I. Benke, and A. Maedche, "The impact of anthropomorphic and functional chatbot design features in enterprise collaboration systems on user acceptance", 14th International Conference on Wirtschaftsinformatik, (2019), 1642-1656.

[48] Hess, T., M. Fuller, and D. Campbell, "Designing interfaces with social presence: Using vividness and extraversion to create social recommendation agents", Journal of the Association for Information Systems 10(12), 2009, pp. 889-919. 